\title{
Is the influence of working memory capacity on high-level cognition mediated by complexity or resource-dependent elementary processes?
}

\author{
Pierre Barrouillet \\ University of Geneva, Geneva, Switzerland \\ RAPHAËLLE LÉPINE \\ University of Bourgogne, Dijon, France \\ AND \\ VALÉRIE CAMOS \\ University of Bourgogne, Dijon, France \\ and Institut Universitaire de France
}

\begin{abstract}
The aim of this study was to investigate the involvement of working memory (WM) in elementary activities and the nature of the mechanisms mediating the influence of WM capacity on high-level cognition. We demonstrate that even elementary activities such as reading digits, subitizing small arrays of dots, or solving simple additions like $3+1$ are sensitive to individual differences in WM capacity. Moreover, we demonstrate that a complex task such as counting large arrays of dots involving these elementary activities as processing steps does not induce WM-related differences beyond what can be predicted from the concatenation of differences elicited by the task's elementary constituents. In line with the time-based resource-sharing model, these results suggest that the influence of WM capacity on high-level cognition is mediated by the impact of a basic general-purpose resource that affects each atomic step of cognition.
\end{abstract}

From the seminal work of Baddeley and Hitch (1974), working memory (WM) has been considered to be a system devoted to the simultaneous maintenance and treatment of information required in high-level cognition. Accordingly, measures of WM capacity such as complex WM spans have proven to be highly predictive of cognitive performance in complex activities (Daneman \& Carpenter, 1980; Kyllonen \& Christal, 1990). The WM span tasks are thus considered to be a way to assess a fundamental capacity required in complex cognition and they are conceived of as measuring a capacity for executive attention and control (Kane, Conway, Hambrick, \& Engle, 2007) or for coordinating multiple-system functioning (Baddeley, 1986). However, two aspects of the relationship between WM capacity and cognition remain undetermined. First, although the involvement of WM in complex activities is widely acknowledged, its implication in the most elementary processes remains unclear. Second, the mechanisms mediating the influence of WM capacity on high-level cognition are largely unknown. The present study addresses these two questions.

According to the time-based resource-sharing model (TBRS; Barrouillet, Bernardin, \& Camos, 2004), the complex activities used as processing components within the traditional WM span tasks, such as reading comprehension, equation solving, or counting, can be analyzed as a series of elementary steps that capture attention for short periods between which attention can be diverted and intermittently switched away for the purpose of maintenance. Indeed, information suffers from a time-related decay when attention is switched away, and frequent refreshing by attentional focusing is needed. This theory assumes that cognitive cost results from the consumption of attentional resources by the most elementary steps of cognition, cognitive load being the proportion of time during which a given activity captures attention.

This theory gave us the rationale for developing a series of WM span tasks in which the processing components consist of a succession of very simple, time-constrained activities, such as reading digits, adding/subtracting 1 or 2 to/from single-digit numbers (the reading digit span and the continuous operation span tasks; Barrouillet et al., 2004), or enumerating small dice-like patterns of dots (Barrouillet, Bernardin, Portrat, Vergauwe, \& Camos, 2007). We selected these activities because they are considered to be elementary. They involve only one cognitive step: the direct retrieval of the answer from memory (Logan, 1988). In line with the TBRS predictions, when performed under

P. Barrouillet, pierre.barrouillet@pse.unige.ch 
time constraints, these elementary activities proved to be as disruptive as complex tasks for concurrent maintenance of information (Lépine, Bernardin, \& Barrouillet, 2005), and our new WM span tasks proved to predict academic achievement better than the traditional reading span and operation span tasks (Lépine, Barrouillet, \& Camos, 2005). These results confirmed the claim of Barrouillet et al. (2004) that cognitive cost is not a matter of complexity per se. Very simple activities consume cognitive resources to the extent that they require attention.

However, two hypotheses, that have not been tested, can be derived from this theory. First, if elementary processes involve a cognitive cost and consume resources because they require attention even for short periods, they should be sensitive to individual differences in WM capacity conceived as the amount of available attention. As far as we know, few studies have directly addressed this question and most often resulted in a negative answer. For example, Tuholski, Engle, and Baylis (2001) observed that highspan individuals were faster than low-span individuals in the complex activity of counting large arrays of dots, but they did not observe any difference in the speed of subitizing. Kane et al. (2007) recently reported that visual search for targets appears to be unrelated to WM capacity. Concerning retrievals from long-term memory, the same authors state that WM capacity appears important for retrieval only when the environment presents a substantial source of interference and competition. By contrast, the TBRS model predicts that any activity involving attention should be sensitive to WM capacity variations.

The second hypothesis concerns the impact of WM capacity on high-level cognition. Usually it is assumed that complex cognitive tasks require WM because they involve the coordination of activities underpinned by multiple components of WM (Baddeley, 1986). By contrast, within the TBRS model, complex activities are nothing more than the concatenation of elementary processes. Thus, the impact of WM would result from the summation of small differences elicited by each attention demanding processing step. As a consequence, complex activities should not induce WM-related differences beyond what could be expected from the differences elicited by their atomic constituents.

The following experiment tested these hypotheses. Adults contrasted on their WM capacity with traditional WM span tasks were presented with the dot counting task already documented by Tuholski et al. (2001). Moreover, the WM-related differences on elementary processes were assessed by presenting participants with elementary numerical activities already used by Barrouillet and colleagues in their WM span tasks such as reading digits, solving very simple additions, and subitizing arrays of 1 to 4 dots. Following Anderson and Lebiere (1998), the TBRS model assumes that these elementary activities, which require retrieval from long-term memory, necessitate the allocation of attention to activate the relevant declarative knowledge above threshold. The higher the amount of attention available, the stronger the activation and the faster the retrieval. Thus, we expected faster responses in individuals with higher WM capacities. Moreover, following
Tuholski et al., for large arrays (5 to 12 dots), we expected high-span individuals to count faster than low-span individuals, but that these differences should be commensurate with and should perfectly reflect those elicited by the elementary activities.

\section{METHOD}

\section{Participants}

Ninety-two undergraduate students from the Universite de Bourgogne ( 76 females, mean age $=20$ years, $S D=3.35$ years) received course credit for participating.

\section{Material and Procedure}

Participants were presented with two WM tasks (the reading span and the alphabet recoding tasks, presented in this order) during a first session, and with three numerical tasks (counting dots, addition solving, and reading digits) that took place in a second session administered 2 weeks later. The order of these three tasks was counterbalanced across participants.

Reading span task. We used the French adaptation of Daneman and Carpenter's (1980) task proposed by Desmette, Hupet, Schelstraete, and Van der Linden (1995). Three blocks of 20 sentences were printed in a booklet with one sentence per page. Each block contained five series of from 2 to 6 sentences presented in ascending order. For each series, participants were asked to successively read aloud the sentences and to remember their final word. At the end of each series, a white page indicated to participants that they had to orally recall these words in the correct order. When the participant failed to correctly recall a given series, the presentation of the block was interrupted and the next block was presented with the same procedure. Before the experiment, participants were presented with two practice series of 2 and 3 sentences, respectively. The span was the total number of words in the experimental series correctly recalled (from 0 to 60 ).

Alphabet recoding task. Alphabet recoding is a task in which participants perform additions and subtractions in the alphabet considered as a numeric chain (e.g., D - $2=\mathrm{B}$; Woltz, 1988). According to Kyllonen and Christal (1990), this task is one of the most accurate measures of WM capacity. Twenty-four random threeletter sets were created and allocated randomly to six operations $(+1,+2,+3,-1,-2,-3)$, each operation receiving 4 sets. Sets in which the result of the operation contained a letter from the initial set (e.g., $\mathrm{AFC}+2=\mathrm{CHE}$ ), sets containing two successive letters (e.g., APB), and sets that could not be calculated (e.g., ZSP + 1) were discarded. The three letters appeared successively on-screen for $1 \mathrm{sec}$, followed by the operation for $2 \mathrm{sec}$. When a question mark appeared, the participants had to perform the mental operations and to calculate the entire response before writing it in a notebook. Sets for which participants paused for more than $1 \mathrm{sec}$ while writing their responses were scored 0 . The 24 sets were presented in a constant order with a break in the middle. The experimental sets were preceded by a 6 -set training session. The score was the number of sets converted correctly (from 0 to 24 ).

Counting dots task. Participants had to evaluate the number of black dots $(0.8 \mathrm{~cm}$ in diameter) randomly displayed in a square frame $15 \mathrm{~cm}^{2}$ at the center of the screen immediately after a 1 -sec ready signal. A voice key stopped the timer when participants gave their response, the array disappeared from the screen, and the experimenter keyed the response to record accuracy. Eight different arrays for each quantity from 1 to 12 were used, resulting in 96 experimental trials presented in a random order. Sixteen training trials preceded the experimental trials.

Addition solving task. Using the same procedure as for the counting task, participants were required to add two digits presented simultaneously on-screen. The digits were displayed in two $5.5 \times$ $5.5 \mathrm{~cm}$ squares placed side by side, separated by a $3.5-\mathrm{cm}$ space. Six experimental trials for each of the 16 possible pairs of digits from 1 to 4 (resulting in 96 trials) were presented in a random order. The 
experimental trials were preceded by 16 practice trials in which all possible pairs were presented once.

Reading digits task. Participants were asked to read aloud Arabic numbers from 1 to 12 appearing on the center of the screen. Each number was shown eight times, resulting in 96 experimental trials presented in random order. Sixteen training trials preceded the experimental trials.

\section{RESULTS}

\section{WM Tasks and Constitution of the Extreme Groups}

Among the 92 participants, the mean score for the alphabet recoding task was $12.10(S D=5.66)$ and the mean reading span was $12.92(S D=5.70)$. The two scores were correlated significantly $(r=.43, p<.001)$. Thus, we calculated a compound WM score by averaging the two $z$ scores. The error rates in the numerical tasks were very low $(0.58 \%, 0.11 \%$, and $0.06 \%$ for the counting dots, the addition, and the reading digit tasks, respectively). The counting dots task was the only task in which the error rate correlated significantly with the WM score $(r=-.32$, $p<.01)$, with participants with lower WM capacities committing more counting errors, mainly in the larger arrays. The main analyses then focused on reaction times. Among the trials with correct responses, those that differed from the mean of the overall sample by more than 3 standard deviations were considered as outliers and were discarded from the analyses (from $1.6 \%$ to $2.5 \%$ of the trials, depending on the task) along with some voice key failures (from $1.8 \%$ to $3.8 \%$ ).

The effect of WM on reaction times was assessed through the entire sample of participants, using linear regressions. To illustrate the magnitude of the effects, we show figures that contrast reaction times in high- and low-span groups. The results from these two groups were also used to investigate the relations among WM-related differences in complex and elementary activities. For this purpose, participants who obtained two positive $z$ scores and a mean $z$ greater than 0.67 constituted the high-WMspan group (18 participants: mean alphabet recoding = $19.39, S D=2.38$; mean reading $\operatorname{span}=20.78, S D=$ 4.98), whereas those who obtained two negative $z$ scores and a mean $z$ lower than -0.67 constituted the low-span group (18 participants: mean alphabet recoding $=5.78$, $S D=3.12$; mean reading span $=7.78, S D=2.58$ ).

\section{Counting Task}

Response times strongly increased with the size of the arrays, from $574 \mathrm{msec}$ for $1 \mathrm{dot}$ to $3,211 \mathrm{msec}$ for 12 dots, with a mean slope relating counting times with the size of the arrays of $268 \mathrm{msec}$. WM capacities had a significant effect on both the mean counting time $[F(1,90)=$ $\left.10.90, p<.01, \eta_{\mathrm{p}}^{2}=.11\right]$ and the slope $[F(1,90)=9.91$, $\left.p<.01, \eta_{\mathrm{p}}^{2}=.10\right]$, participants with lower WM capacities being slower and exhibiting a steeper slope. The pattern of response times conformed with the classical bent curve indicating the use of subitizing and counting in both highand low-span participants (Figure 1). Subitizing range was computed for the entire sample using the method of

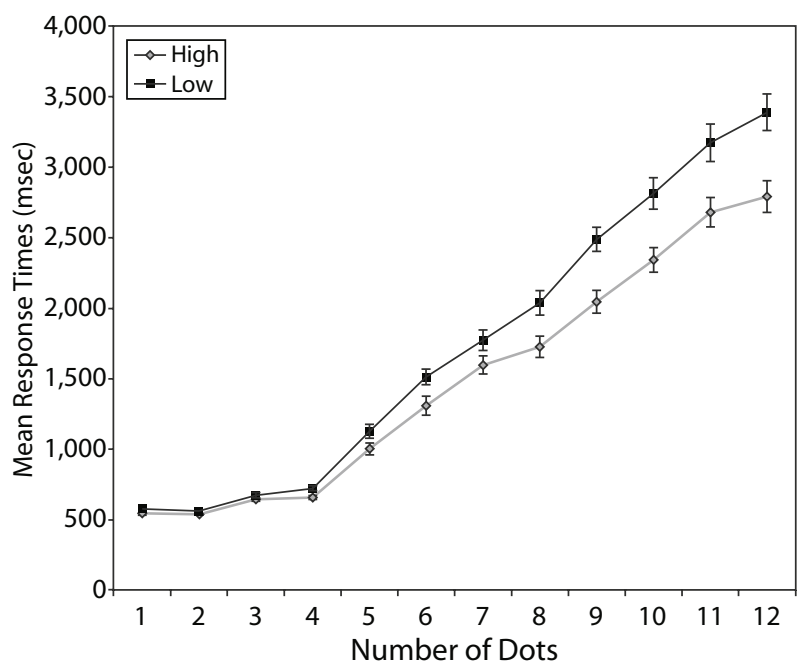

Figure 1. Mean enumeration times (in milliseconds) as a function of the group (high- and low-span participants) and the number of dots.

the incremental quadratic trend analysis as in Tuholski et al. (2001), revealing a range of 4 and, consequently, a counting range from 5 upward.

In this last range (sizes 5-12), WM spans had a significant effect on counting times that were shorter in participants with higher WM capacities $[F(1,90)=10.60, p<.01$, $\left.\eta_{\mathrm{p}}^{2}=.11\right]$. As observed by Tuholski et al. (2001), the effect of WM capacities increased with the quantities from 5 to 12 , with lower working memory capacities resulting in steeper slopes $\left[F(1,90)=8.51, p<.01, \eta_{\mathrm{p}}^{2}=.09\right]$ (Table 1$)$.

\section{Elementary Tasks}

Subitizing times were assessed on the arrays up to 4 . The mean response times were 574, 550, 661, and $699 \mathrm{msec}$ for $1,2,3$, and 4 dots, respectively, with a mean slope of $49 \mathrm{msec}$. As we predicted, higher WM capacities were related with faster subitizing $[F(1,90)=6.66, p=.01$, $\left.\eta_{\mathrm{p}}^{2}=.07\right]$. This effect did not interact with the size of the arrays, WM capacities having no significant effect on the subitizing slope $\left[F(1,90)=2.09, p=.15, \eta_{\mathrm{p}}^{2}=.02\right]$.

Consistent with our predictions, higher WM capacities were related with faster responses in addition solving $\left[F(1,90)=15.64, p<.001, \eta_{\mathrm{p}}^{2}=.15\right]$. As usually reported in the literature, we observed a size effect, with slower responses for larger additions. The slope of the regression line between the sum and the response times was significantly different from zero in the entire sample $($ mean $=16 \mathrm{msec}, S D=14 \mathrm{msec})[t(91)=10.93, p<$ $.001]$. Interestingly, this slope was steeper in participants with lower WM spans $\left[F(1,90)=12.91, p<.001, \eta_{\mathrm{p}}^{2}=\right.$ .13] (Figure 2), indicating larger WM-related differences for larger problems.

Finally, higher WM capacities were also related to faster reading of numbers $\left[F(1,90)=9.37, p<.01, \eta_{\mathrm{p}}^{2}=\right.$ .09]. There was also a significant effect of the size of the number to be read, but it should be noted that this was not a size effect; large numbers did not take longer to read 
Table 1

Correlations Among Response Times in Counting, Subitizing, Addition Solving, Reading Digits, Slopes of Counting and Subitizing, Size Effect in Addition Solving, and WM Score

\begin{tabular}{lccccccc}
\hline & $\begin{array}{c}\text { Counting } \\
\text { Slope }\end{array}$ & Addition & $\begin{array}{c}\text { Addition } \\
\text { Size Effect }\end{array}$ & $\begin{array}{c}\text { Reading } \\
\text { Digits }\end{array}$ & Subitizing & $\begin{array}{c}\text { Subitizing } \\
\text { Slope }\end{array}$ & $\begin{array}{c}\text { WM } \\
\text { Score }\end{array}$ \\
\hline Counting & $.852^{* *}$ & $.610^{* *}$ & $.333^{* *}$ & $.484^{* *}$ & $.623^{* *}$ & .228 & $-.325^{* *}$ \\
Counting slope & & $.359^{* *}$ & .125 & $.294^{* *}$ & $.363^{* *}$ & .042 & $-.294^{* *}$ \\
Addition & & & $.602^{* *}$ & $.739^{* *}$ & $.772^{* *}$ & .217 & $-.385^{* *}$ \\
Addition size effect & & & & $.390^{* *}$ & $.359^{* *}$ & .099 & $-.354^{* *}$ \\
Reading digits & & & & & $.783^{* *}$ & .084 & $-.307^{* *}$ \\
Subitizing & & & & & & $.322^{* *}$ & $-.263^{*}$ \\
Subitizing slope & & & & & & & -.151 \\
\hline
\end{tabular}

${ }^{*} p<.05 . \quad{ }^{* *} p<.01$.

than small numbers, the mean slope being practically nil $(-3 \mathrm{msec})$ and not related to working memory capacities $\left[F(1,90)=2.26, p=.14, \eta_{\mathrm{p}}^{2}=.02\right]$ (Figure 3 ).

The pattern of correlations revealed strong relationships between subitizing, additions, and reading digits, as their common reliance on WM capacities would lead one to expect. Interestingly, the subitizing slope that proved to be independent from WM was also poorly related to other measures (Table 1).

\section{Differences on Complex and Elementary Processes}

According to the TBRS model, complex activities are nothing more than the concatenation of elementary steps of cognition. WM-related differences in complex activities should result from the summation of small differences elicited by each attention-demanding processing step. If this hypothesis is correct, WM differences in complex activities should be perfectly commensurate with those observed in elementary activities, and knowing the size of the latter should make it possible to predict the size of the former within a perfectly additive linear model. By contrast, if differences in complex tasks result from peculiar and demanding processes, such as coordination of activities underpinned by multiple components, they should induce greater WM-related differences than those elicited by elementary processes.

To test these hypotheses, we first assessed the WMrelated differences in elementary processes. We plotted the mean response times in the low-span group against those in the high-span group for each item in each task involving elementary processes (i.e., the 12 items of the reading digit task, the 4 sizes of arrays in the subitizing range, and the 16 additions). Despite strong differences in nature between the tasks and the reduced range of times that they elicited (from 400 to $850 \mathrm{msec}$ ), this analysis revealed a clear linear trend, with an $r$ value of .989, a slope of 1.252, and an intercept of $-84 \mathrm{msec}$ (Figure 4). The slope higher than 1 indicates that WM-related differences increased steadily with increases in the duration of the activities. These parameters were used to predict the WMrelated differences that should be observed in the counting task (range, 5-12) if, as we hypothesized, differences in complex activities do not go beyond those observed in

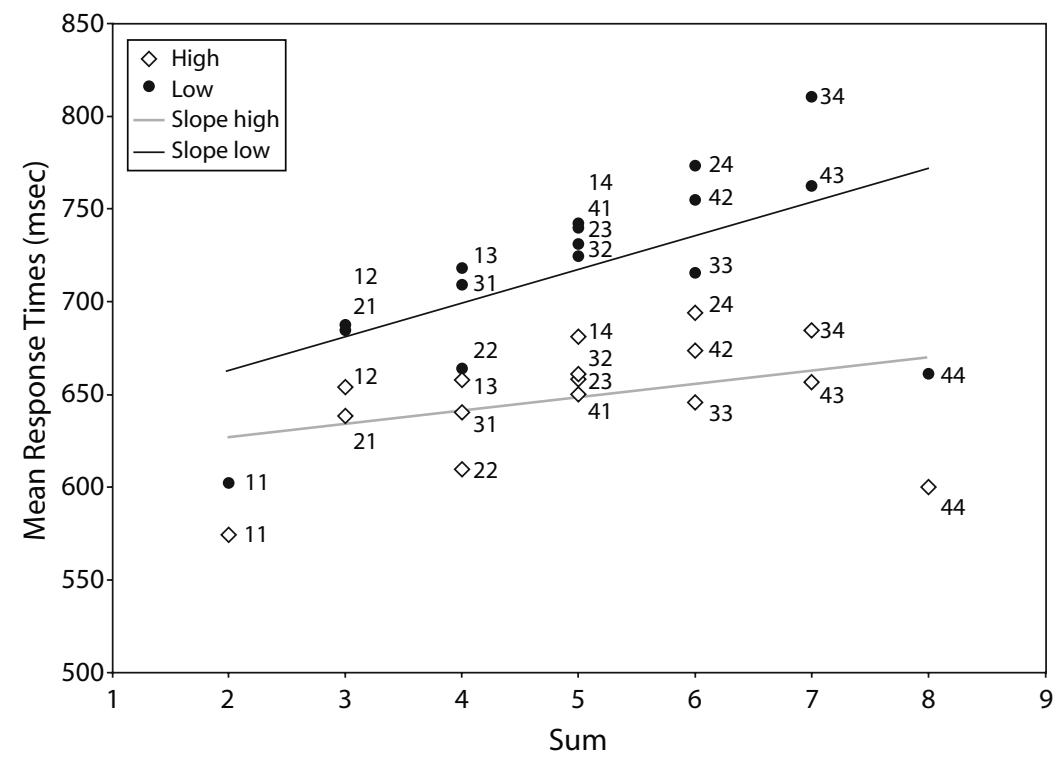

Figure 2. Mean response times (in milliseconds) for the addition solving as a function of the group (high- and low-span participants) and the sum of the two operands. 


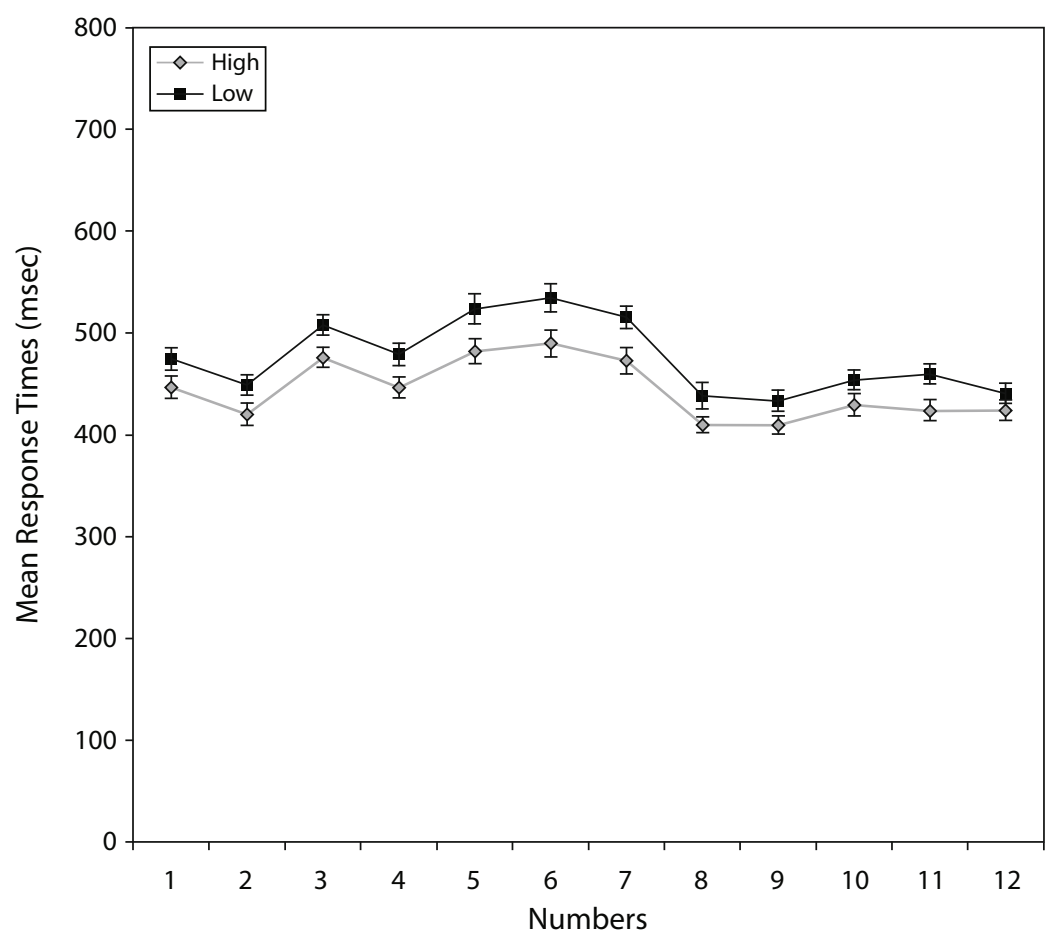

Figure 3. Mean reading times (in milliseconds) of digits as a function of the group (high- and low-span participants) and the presented number.

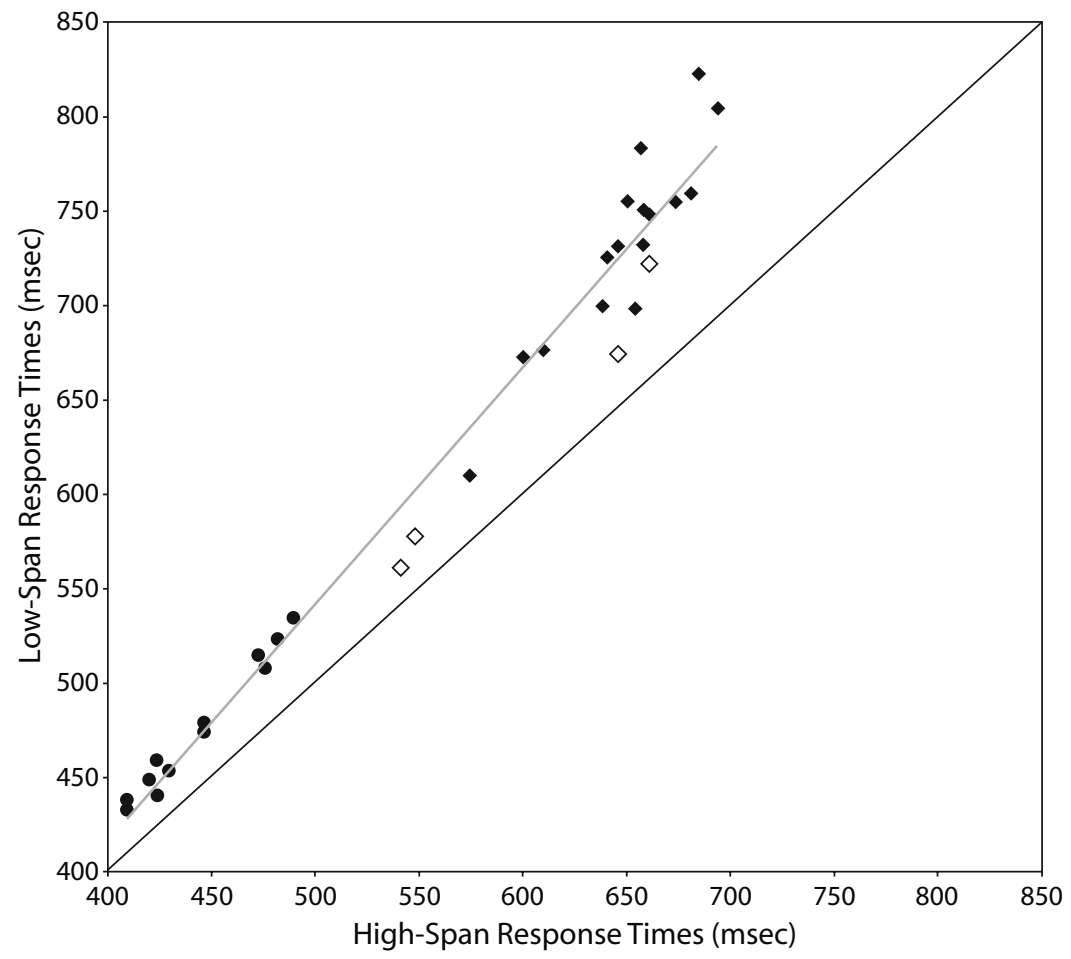

Figure 4. Relationship between mean response times in high- and low-span groups for the items assumed to involve elementary processes, with regression slope shown (black dots for the reading digit task, white diamonds for subitizing, and black diamonds for addition). 
elementary processes. In this case, when one is plotting the mean response times in the low-span group against those in the high-span group for the complex activity of counting, the resulting dots should fall on the line resulting from the regression analysis on elementary processes. For this purpose, predicted times for the low-span group were calculated from the observed response times in the high-span group, using the linear function provided by the elementary processes analysis (Figure 5).

Our hypothesis was confirmed. The fit between the observed and predicted values for the low-span group was nearly perfect, with $99.7 \%$ of the variance explained. Each predicted value fell within the $95 \%$ confidence interval for the corresponding observed mean in the lowspan group. The predicted and observed linear functions had practically identical slopes (1.252 and 1.258 , respectively) and differed only in their intercepts $(-84 \mathrm{msec}$ and $-140 \mathrm{msec}$, respectively). This difference was due to the fact that observed differences on complex activities were even slightly smaller than those predicted from elementary processes (most of the observed values fall under the line of predicted values in Figure 5). Thus, as we predicted, the differences observed in counting were absolutely proportionate to those elicited by elementary activities. ${ }^{1}$

\section{DISCUSSION}

Two main findings arose from this experiment. First, individual differences in WM capacity affect elemen- tary processes, high-span individuals being faster in solving small additions, subitizing, and reading digits. This result confirms one of the main assumptions of the TBRS model: Any process that occupies attention for a sizeable portion of time, elementary though it may be, involves a cognitive cost and is thus sensitive to variations in WM capacity.

Second, a complex activity such as counting does not yield WM-related differences beyond what can be expected from the differences observed in the elementary components of this activity. Counting in adults has been described as relying on successive additions of small quantities that can be subitized (Aoki, 1977). We have seen that these atomic components are sensitive to variations in WM capacity. Of course, it could be assumed that, to avoid double-counting and omissions, one must keep track of objects already counted, and that high-span individuals differ probably from low-span individuals on this controlled activity (Tuholski et al., 2001). However, our study has demonstrated that the differences between high- and low-span individuals on the complex activity of counting are exactly proportionate to those observed on its elementary constituents.

Such a finding is compatible with the TBRS model, which assumes a sequential functioning of WM. The time needed to perform each processing step would depend on a basic general capacity, conceived as the amount of available attention needed to activate relevant items of knowledge and procedures. Individual differences in this

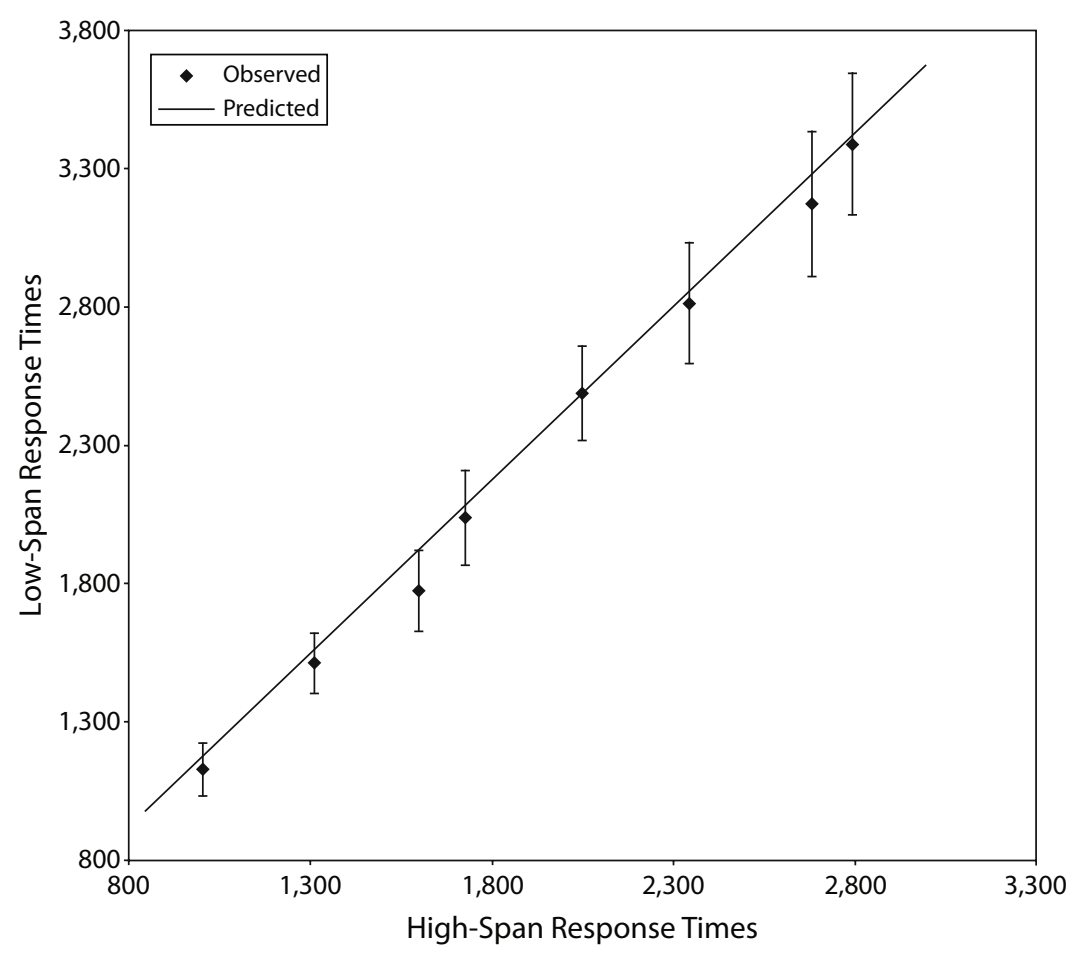

Figure 5. Observed counting times (array sizes from 5 to 12) for the low- (with $\pm 2 S E$ error bars) and high-span groups, with linear function of the predicted values in the low-span group under the hypothesis that WM-related differences in counting follow the same function as do the differences observed in elementary processes. 
basic capacity would result in small differences in the time needed to perform each attention-demanding processing step. Thus, exactly as we observed, the difference in response time between high- and low-WM-capacity individuals would steadily increase as the task involves the concatenation of more processing steps and takes longer.

To conclude, because WM is the central and executive system of the cognitive architecture, its capacity should and does affect the most elementary processes on which cognition is based. However, this is not to say that all aspects of cognition are sensitive to WM capacity. Our theory assumes that the effects reported in this study should be restricted to processes that require attention. For example, we have already shown that simple reactions do not involve WM (Barrouillet et al., 2007). Drawing up the map of the WM-(in)dependent processes is probably one of the imperative tasks in the agenda of WM researchers.

\section{AUTHOR NOTE}

Part of this research was done when the last author was invited fellow at University of Bristol, funded by the Leverhulme Trust, and was also supported by Grants 05 -BLAN-0346-01 from the ANR. Correspondence concerning this article should be addressed to P. Barrouillet, Universite de Genève, FPSE, 40, bd du Pont d'Arve, 1205 Geneva (e-mail: pierre .barrouillet@pse.unige.ch).

\section{REFERENCES}

Anderson, J. R., \& Lebiere, C. (1998). Atomic components of thought. Mahwah, NJ: Erlbaum.

AOKI, T. (1977). On the counting process of patterned dots. Tohoku Psychologica Folia, 36, 15-22.

BADDELEY, A. D. (1986). Working memory. Oxford: Oxford University Press, Clarendon Press.

Baddeley, A. D., \& Hitch, G. (1974). Working memory. In G. A. Bower (Ed.), The psychology of learning and motivation (Vol. 8, pp. 647-667). New York: Academic Press.

Barrouillet, P., Bernardin, S., \& Camos, V. (2004). Time constraints and resource sharing in adults' working memory spans. Journal of Experimental Psychology: General, 133, 83-100.

Barrouillet, P., Bernardin, S., Portrat, S., Vergaume, E., \& CAmos, V. (2007). Time and cognitive load in working memory. Journal of Experimental Psychology: Learning, Memory, \& Cognition, 33, 570-585.

Daneman, M., \& Carpenter, P. A. (1980). Individual differences in working memory and reading. Journal of Verbal Learning \& Verbal Behavior, 19, 450-466.
Desmette, D., Hupet, M., Schelstraete, M. A., \& VAn der LinDEN, M. (1995). Adaptation en langue française du "Reading Span Test” de Daneman et Carpenter (1980). L'Année Psychologique, 95, 459-482.

Kane, M. J., Conway, A. R. A., Hambrick, D. Z., \& Engle, R. W. (2007). Variation in working memory capacity as variation in executive attention and control. In A. R. A. Conway, C. Jarrold, M. J. Kane, A. Miyake, \& J. N. Towse (Eds.), Variation in working memory (pp. 21-46). New York: Oxford University Press.

Kyllonen, P. C., \& Christal, R. E. (1990). Reasoning ability is (little more than) working-memory capacity? Intelligence, 14, 389-433.

LÉPine, R., Barrouillet, P., \& CAmos, V. (2005). What makes working memory spans so predictive of high-level cognition? Psychonomic Bulletin \& Review, 12, 165-170.

Lépine, R., Bernardin, S., \& Barrouillet, P. (2005). Attention switching and working memory spans. European Journal of Cognitive Psychology, 17, 329-345.

Logan, G. D. (1988). Toward an instance theory of automatization. Psychological Review, 95, 492-527.

Ratcliff, R., Spieler, D., \& McKoon, G. (2004). Analysis of group differences in processing speed: Where are the models of processing? Psychonomic Bulletin \& Review, 11, 755-769.

Tuholski, S. W., Engle, R. W., \& Baylis, G. C. (2001). Individual differences in working memory capacity and enumeration. Memory \& Cognition, 29, 484-492.

Woltz, D. J. (1988). An investigation of the role of working memory in procedural skill acquisition. Journal of Experimental Psychology: General, 117, 319-331.

\section{NOTE}

1. The use of Brinley plots to assess between-group differences in processing has been the object of some criticisms. Ratcliff, Spieler, and McKoon (2004) noted that, although it is statistically inappropriate to use standard regression for estimating the slope of Brinley plots, it is likely not to be a severe problem if the correlation is high (the correlation that we observed was .989). However, when the value of the slope is to be used for hypothesis testing, as was the case here, they suggest that one use the ratio of the $S D$ s of the $x$ and $y$ values, because standard linear regression underestimates the true slope when there is error in the $x$ values. In our data, this ratio was very close to the slope of the regression line (1.268 vs. 1.252). Moreover, it is worth noting that underestimating the true slope of the function on elementary processes could only run counter to our hypothesis, which is that the observed values on complex activities would not exceed the predicted values. Actually, even if the slope was underestimated, the observed values were slightly lower (Figure 5).

(Manuscript received July 2, 2007; revision accepted for publication November 6, 2007.) 